\title{
Addressing the Third Delay in Saving Mothers, Giving Life Districts in Uganda and Zambia: Ensuring Adequate and Appropriate Facility-Based Maternal and Perinatal Health Care
}

Diane Morof, ${ }^{a, b}$ Florina Serbanescu, ${ }^{a}$ Mary M. Goodwin, ${ }^{a}$ Davidson H. Hamer, ${ }^{c, d}$ Alice R. Asiimwe, ${ }^{e}$ Leoda Hamomba, ${ }^{f}$ Masuka Musumali, ${ }^{9}$ Susanna Binzen, ${ }^{a}$ Adeodata Kekitiinwa, ${ }^{e}$ Brenda Picho,

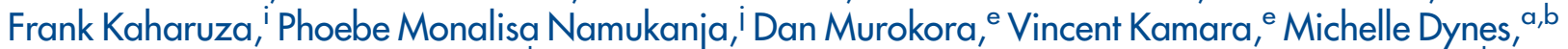
Curtis Blanton, ${ }^{a}$ Agnes Nalutaaya, ${ }^{\text {h }}$ Fredrick Luwaga, ${ }^{e}$ Michelle M. Schmitz, ${ }^{a}$ Jonathan LaBrecque, ${ }^{k}$ Claudia Morrissey Conlon, 'Brian McCarthy, ${ }^{\mathrm{m}}$ Charlan Kroelinger, ${ }^{a}$ Thomas Clark, ${ }^{a}$ on behalf of the Saving Mothers, Giving Life Working Group

Saving Mothers, Giving Life used 6 strategies to address the third delay-receiving adequate health care after reaching a facility-in maternal and newborn health care. The intervention approaches can be adapted in low-resource settings to improve facility-based care and reduce maternal and perinatal mortality.

\section{ABSTRACT}

Background: Saving Mothers, Giving Life (SMGL) is a 5-year initiative implemented in participating districts in Uganda and Zambia that aimed to reduce deaths related to pregnancy and childbirth by targeting the 3 delays to receiving appropriate care: seeking, reaching, and receiving. Approaches to addressing the third delay included adequate health facility infrastructure, specifically sufficient equipment and medications; trained providers to provide quality evidence-based care; support for referrals to higher-level care; and effective maternal and perinatal death surveillance and response.

Methods: SMGL used a mixed-methods approach to describe intervention strategies, outcomes, and health impacts. Programmatic and monitoring and evaluation data-health facility assessments, facility and community surveillance, and population-based mortality studies - were used to document the effectiveness of intervention components.

Results: During the SMGL initiative, the proportion of facilities providing emergency obstetric and newborn care (EmONC) increased from $10 \%$ to $25 \%$ in Uganda and from $6 \%$ to $12 \%$ in Zambia. Correspondingly, the delivery rate occurring in EmONC facilities increased from $28.2 \%$ to $41.0 \%$ in Uganda and from $26.0 \%$ to $29.1 \%$ in Zambia. Nearly all facilities had at least one trained provider on staff by the

a Division of Reproductive Health, U.S. Centers for Disease Control and Prevention, Atlanta, GA, USA.

b U.S. Public Health Service Commissioned Corps, Rockville, MD, USA.

'Department of Global Health, Boston University School of Public Health, Boston, MA, USA.

d Section of Infectious Diseases, Department of Medicine, Boston Medical Center, Boston, MA, USA.

e'Baylor College of Medicine Children's Foundation-Uganda, Kampala, Uganda. ${ }^{f}$ Division of Global HIV and TB, U.S. Centers for Disease Control and Prevention, Lusaka, Zambia.

gFamily Health Division, U.S. Agency for International Development, Lusaka, Zambia.

h Infectious Diseases Institute, College of Health Sciences, Makerere University, Kampala, Uganda.

iHIV Health Office, U.S. Agency for International Development, Kampala, Uganda. i Division of Global HIV and TB, U.S. Centers for Disease Control and Prevention, Kampala, Uganda.

kBureau for Global Health, U.S. Agency for International Development Washington DC. Now with Boston Children's Hospital, Boston, MA, USA

'Bureau for Global Health, U.S. Agency for International Development,

Washington, DC, USA.

${ }^{m}$ Eck Institute for Global Health, University of Notre Dame, Notre Dame, IN, USA.

Correspondence to Diane Morof (dmorof@cdc.gov). endline evaluation. Staffing increases allowed a higher proportion of health centers to provide care 24 hours a day/7 days a week by endline-from $74.6 \%$ to $82.9 \%$ in Uganda and from $64.8 \%$ to $95.5 \%$ in Zambia. During this period, referral communication improved from $93.3 \%$ to $99.0 \%$ in Uganda and from $44.6 \%$ to $100 \%$ in Zambia, and data systems to identify and analyze causes of maternal and perinatal deaths were established and strengthened.

Conclusion: SMGL's approach was associated with improvements in facility infrastructure, equipment, medication, access to skilled staff, and referral mechanisms and led to declines in facility maternal and perinatal mortality rates. Further work is needed to sustain these gains and to eliminate preventable maternal and perinatal deaths.

\section{INTRODUCTION}

aving Mothers, Giving Life (SMGL) is a 5-year initiative designed to reduce deaths related to pregnancy and childbirth. SMGL used a coordinated approach targeting the 3 delays-seeking, reaching, and receiving 
adequate care-that contribute to maternal deaths. ${ }^{1}$ This article focuses on maternal and perinatal deaths due to the third delay, the lack of receipt of timely, adequate, and appropriate obstetric care at a health care facility (Figure). ${ }^{2}$ An estimated $75 \%$ of maternal deaths globally result from direct obstetric causes, with more than half attributed to hemorrhage, hypertensive disorders, and sepsis. ${ }^{3}$ Moreover, approximately $29 \%$ of newborn deaths in sub-Saharan Africa are attributed to intrapartum-related events. ${ }^{4}$ Facility-based maternal and newborn care, including access to skilled providers and neonatal resuscitation, improves the likelihood of maternal and infant survival. ${ }^{5,6}$ Although multiple socioeconomic and environmental factors affect maternal and neonatal survival, reducing the delay in receiving adequate and appropriate care at a health facility is key to improving health outcomes. ${ }^{7}$

Reducing deaths related to the third delay requires overcoming barriers to timely, adequate, and appropriate obstetric and neonatal care in facilities. $^{7-9}$ Many deaths are largely preventable if providers and facilities use the 9 evidence-based medical interventions termed 'signal functions' that comprise emergency obstetric and newborn care (EmONC). ${ }^{10}$ Facilities may be classified as providing basic EmONC (BEmONC) if they are able to (1) administer parenteral antibiotics, (2) administer uterotonic drugs for active management of the third stage of labor and prevention and management of postpartum hemorrhage, (3) use parenteral anticonvulsants for the prevention and management of pre-eclampsia/eclampsia, (4) perform manual removal of placenta, (5) perform removal of retained products, (6) perform assisted vaginal delivery, and (7) perform neonatal resuscitation; and classified as providing comprehensive EmONC (CEmONC) if they are able to perform the 7 basic signal functions plus being able to perform a (8) cesarean section and (9) blood transfusion. ${ }^{6,10}$

\section{Barriers and Interventions to Improve the Third Delay}

Interventions to address the third delay and ensure timely access to the 9 signal functions

FIGURE. Context of Quality of Health Services for the Third Delay

\section{Contributing Factors \\ (Distal Determinants)}

\begin{tabular}{|l|l|}
\hline $\begin{array}{l}\text { Economic and } \\
\text { political policies }\end{array}$ & $\begin{array}{l}\text { Indirect household level: } \\
\text { Lulture } \\
\text { Religion socioeconomic status, } \\
\text { Sociocultural norms of medical } \\
\text { interventions, HIV test outcome } \\
\text { stigma, low family support, low } \\
\text { exposure to heath care } \\
\text { messages, and low status of } \\
\text { women }\end{array}$ \\
\hline $\begin{array}{l}\text { Indirect health system level: } \\
\text { Inadequate formal medical } \\
\text { training, outdated clinical } \\
\text { guidelines, lack of continued } \\
\text { training for health workers, lack } \\
\text { of respectful care, low wages for } \\
\text { health staff, poor hospital } \\
\text { conditions, lack of supervision, } \\
\text { lack of motivation, and low } \\
\text { quantity and quality of } \\
\text { information education and } \\
\text { communication materials }\end{array}$ \\
\hline
\end{tabular}

Underlying Factors

(Proximal Determinants)

\section{Direct household and} individual level:

Lack of funds for health care and resistance to certain interventions due to cost or sociocultural factors

Direct health system level: Staff shortages, long wait time, inadequate 24-hour availability, lack of proper medical storage, use of less effective interventions, lack of emergency transport and logistics for referral, cost of care, and lack of essential medicines, equipment, and blood supplies 
have concentrated primarily on health facility care during the critical period of labor, delivery, and first 24 hours postpartum when most maternal deaths and about half of newborn deaths occur. $^{11,12}$ Effective interventions ensure the availability of skilled health providers, sufficient and appropriate medical commodities and equipment, accessible high-quality obstetric care, and high-functioning inter-facility referral and receiving processes. ${ }^{7,13}$

Human resource shortage is the most frequently cited factor associated with lack of appropriate care in health facilities; it encompasses inadequate training, lack of access to continuing education, staffing shortages, lack of motivation due to poor working conditions and low pay, and lack of optimal supervision and management. ${ }^{7,14}$ Lack of appropriate medical commodities and equipment is the second most commonly referenced challenge to reducing the third delay, with inadequate drug supply, lack of equipment, and lack of blood cited as common problems. ${ }^{15}$

Low demand for facility deliveries and other obstetric services may occur for many reasons, including in low population density areas where there is a need to invest in the availability and accessibility of obstetric care. ${ }^{16}$ Prior negative experiences with unclean, unsafe, or disrespectful care; a lack of competent providers; real or perceived high costs of health care; and cultural beliefs and practices can also contribute to low facility use. ${ }^{17-22}$ While, the third delay is most directly associated with facility characteristics and quality of care, certain aspects of individual and household barriers, including the negative experiences described above, may contribute to delays in receiving appropriate facility care. Additionally, while delays associated with poor referral practices directly contribute to the second delay, they also contribute to the third delay when facilities delay referral to higher levels or incorrectly refer patients to facilities that cannot provide the level of care needed. Finally, delays in receiving care after arrival at the health care facility contribute to the third delay. ${ }^{23-25}$ When health facilities have sufficient beds, essential drugs, medical equipment, robust infrastructure, skilled care, and consistent operating hours, women and newborns are more likely to receive appropriate facility care. $^{26,27}$

\section{SMGL Context in Relation to the Third Delay}

In Uganda, at the beginning of the SMGL initiative in $2011,57 \%$ of all births occurred in a health facility. ${ }^{28}$ Facility births were more common in urban areas $(90 \%)$ than in rural areas $(52 \%)$, as were cesarean deliveries (13.7\% of urban births, $3.9 \%$ of rural births). ${ }^{28}$ The 4 contiguous SMGL districts-Kabarole, Kibaale, Kamwenge, and Kyenjojo-were predominantly rural, with an average population density of 26.2 women of reproductive age per square kilometer and a facility delivery rate of $45.5 \%$, which was slightly lower than the national average of $55 \% .^{28}$ At the time the initiative began, national reports found that most health centers in the SMGL Uganda districts had inadequate infrastructure for maternity units, too few functional operating theatres, insufficient numbers of skilled providers, poorly documented health care services and outcomes, and low-functioning referral and communication systems. $^{29}$

In Zambia, $67 \%$ of births in 2013 took place in health facilities. ${ }^{30}$ In contrast to Uganda, the Zambia SMGL districts were more sparsely populated, with 3.9 women of reproductive age per square kilometer, requiring longer-distance travel to care. ${ }^{30}$ Cesarean section rates were low in all 3 provinces-3.7\% in Eastern Province, 2.9\% in Southern Province, and 3.0\% in Luapulareflecting lower rates in rural $(3.0 \%)$ compared with urban areas (7.2\%). ${ }^{30}$ In 2010, 2 of the 3 SMGL districts in Luapula and Eastern provinces were among the 5 provinces with the highest maternal mortality rates and the 3 provinces with the highest child mortality rates. ${ }^{31}$ Poor coverage of maternal and neonatal health services in Zambia was attributed, in part, to weak referral systems, the absence of systems to handle obstetric and neonatal health emergencies, and poor logistics to manage essential drugs. $^{31}$

This article highlights SMGL interventions related to reducing the third delay by ensuring that women and newborns received adequate and appropriate care once at a health care facility. We describe intervention approaches and results in 6 areas in health care facilities necessary to address the third delay:

1. Adequate infrastructure to provide EmONC

2. Sufficient medical supplies, equipment, and medications

3. Sufficient trained health care providers at facilities

4. Improved quality of care and care that is evidence-based

5. Referral capacity to support transfers to higher-level care
Human resource shortage is the most frequently cited factor associated with lack of appropriate care in health facilities.

SMGL

interventions related to reducing the third delay addressed 6 key areas for improvement: infrastructure, medical supplies and medication, health care provider training, evidence-based care, referrals, and maternal and perinatal surveillance. 
6. Effective maternal and perinatal health surveillance

Although specific programmatic interventions, detailed in Table 1, varied by location, the overall approaches were aligned.

\section{METHODS}

SMGL used both quantitative and qualitative methods to describe implementation of intervention strategies, outcomes, and health impacts. SMGL implementing partners collected programmatic data throughout the initiative. Partners increased efforts during the initiative to coordinate monitoring and evaluation and harmonize data collection to understand which intervention components were effective. Programmatic interventions detailed here principally occurred in Phase 1 and continued into Phase 2, further details on the content of the phases are described elsewhere. ${ }^{32}$ To evaluate the overall impact of the SMGL initiative, we compared data collected at baseline-the 12 months prior to the onset of the initiative, June 2011 to May 2012-with data collected at the endline, January to December 2016. We collected supplemental qualitative data to describe the influence of specific maternal and perinatal interventions on improving appropriate facility-based care.

\section{Quantitative Data and Analytic Methods}

We used health facility assessments (HFAs), facilitybased outcome monitoring, and community-based surveillance to capture key intervention outcomes and health impacts. Approaches and methods for each of these data sources are described in depth. ${ }^{32}$ For our study, we compared maternal and perinatal data collected at baseline and endline.

\section{Health Facility Assessments}

SMGL partners implemented HFAs in SMGL districts $^{35}$ to assess changes in facility functionality, including facility infrastructure; transportation and communications referral practices; capacity to perform EmONC; equipment and supplies, including essential medicines; staffing, training, and 24-hour availability of medical staff in health facilities; and selected aspects of respectful care. A total of 105 and 110 facilities were assessed at baseline and endline in Uganda and Zambia, respectively. Indicators derived from HFAs that were used in this analysis include basic facility infrastructure and staffing, promotion of protocols and guidelines, availability of essential drugs, performance of EmONC signal functions, and performance of maternal death reviews. Definitions and descriptions of indicators of interest are included in Box 1.

\section{Facility-Based Outcome Data}

Facility-based pregnancy outcome data collection captured clinical data on procedures, complications, and health outcomes. This analysis uses percent of deliveries in EmONC facilities derived from the facility-based data sources.

\section{Population-Based Data}

We calculated the proportion of deliveries in EmONC facilities with number of live births as the denominator. In Zambia, at baseline, districtspecific population from the 2010 national census, external district-specific growth rates, and crude birth rates were used to estimate the number of live births in the SMGL districts. ${ }^{33,34}$ At endline, the number of live births was determined by applying district-specific facility delivery rates calculated from the 2017 SMGL census. ${ }^{32}$ In Uganda, for both baseline and endline, population statistics were derived from district-wide SMGL household enumerations conducted in 2012 and 2017 in conjunction with the Reproductive Age Mortality Survey studies. ${ }^{32}$

HFAs and pregnancy outcomes monitoring were conducted in virtually all facilities that provide maternity care in SMGL districts. Because we considered data to be complete counts rather than a sample and reported indicators as percentages, our data were not subject to sampling error. We calculated the z-statistic using the McNemar's test for dichotomous responses for matched pairs of data at baseline and endline periods, and calculated relative change in indicators by subtracting the baseline value from endline value and dividing by the baseline value.

\section{Qualitative Data and Analytic Methods}

Qualitative data sources included Phase 1 and Phase 2 project reports, documents submitted by SMGL implementing partners, and special qualitative studies focused on determining the effectiveness of the interventions.

In November 2017, we conducted 16 individual or small group (up to 3 people) in-depth interviews with 28 maternal and newborn health providers from 15 health centers and hospitals in SMGL districts in Uganda. In most cases, the head of maternity identified the most appropriate individuals to participate based on their experience with the Birth Weight and Age-at-Death Boxes for Interv- 
TABLE 1. SMGL Interventions to Reduce the Third Delay, 201 1-2016

Country-Specific Interventions ${ }^{a}$

Strategies and Approaches

Uganda

Zambia

Strategy 1. Ensure facilities have adequate infrastructure to provide EmONC

Approach 1.1: Support expansion and renovation of operating theaters and facility enhancements to accommodate additional deliveries

- Renovated and upgraded operating theaters

- Increased the size of labor rooms

- Provided additional delivery beds to allow more women to deliver in facilities and stay longer postpartum

Approach 1.2: Support facility enhancements to improve neonatal survival

- Procured incubators, infant warmers, and phototherapy lamps

- Renovated infrastructure to have designated space for KMC and to create NICUs

Approach 1.3: Support improved

- Provided safe water systems at health facilities access to electricity and water
- Provided solar panels at facilities to improve continuity of access to electricity and light
- Supported renovation of birthing centers, delivery rooms, and maternity annexes

- Provided additional delivery beds to allow more women to deliver in facilities and stay longer postpartum

- Refurbished dedicated KMC rooms at hospitals

- Improved lighting systems for delivery rooms

- Improved piped water to maternity annexes

\section{Strategy 2. Ensure sufficient medical supplies, equipment, and medications}

Approach 2.1: Strengthen supply chains for essential supplies and medicines
- Procured essential medication and backup supply of commodities for all sites on the SMGL project

- Redistributed supplies between health facilities to reduce stock-outs

- Implemented SMS reminder system to ensure timely drug ordering

- Equipped health centers with BEmONC equipment and supplies

Approach 2.2: Strengthen availability of blood supplies and surgical equipment
- Strengthened and maintained the blood supply system in CEmONC sites and supported new regional blood bank

- Provided new blood refrigerators

- Procured and distributed new surgical equipment to facilities
- Procured essential emergency medications and supplies with backup

- Trained staff in eLMIS

- Equipped health centers with BEmONC equipment and supplies

- Assembled and distributed uterine balloon tamponade kits, and CPAP machines

- Procured and distributed centrifuges, refrigerators, and freezers to support blood bank

- Procured and distributed new surgical equipment to facilities

\section{Strategy 3. Ensure sufficient trained health care providers at facilities}

\begin{tabular}{llll}
\hline Approach 3.1: Recruited staff & - $\begin{array}{l}\text { Recruited new medical doctors and nurse/midwives } \\
\text { through a joint hiring process with the districts }\end{array}$ & - Recruited new nurse/midwives \\
\hline $\begin{array}{ll}\text { Approach 3.2: Trained health profes- } \\
\text { sionals in emergency obstetric care, }\end{array}$ & $\begin{array}{l}\text { Trained medical officers, anesthetic officers, and } \\
\text { including obstetric surgeries }\end{array}$ & $\begin{array}{l}\text { midwives/nurses in CEmONC } \\
\text { - Conducted surgical skills course for medical offi- } \\
\text { cers, including decision making and caesarean } \\
\text { section }\end{array}$ & $\begin{array}{l}\text { Trained doctors, nurses, midwives, and } \\
\text { anesthetists in EmONC, clinical decision } \\
\text { making, obstetric complications, hemor- } \\
\text { rhage management with uterine balloon } \\
\text { tamponade, early HBB, and CPAP }\end{array}$ \\
& $\begin{array}{l}\text { Trained providers on neonatal resuscitation/HBB } \\
\text { and used drills to reinforce lessons }\end{array}$ & $\begin{array}{l}\text { Limited rotation of trained providers to dif- } \\
\text { ferent wards } \\
\text { Supported capacity building of laboratory } \\
\text { staff for blood services }\end{array}$
\end{tabular}


TABLE 1. Continued

Country-Specific Interventions ${ }^{a}$

\section{Strategies and Approaches}

Approach 3.3: Provided mentoring and supportive supervision to newly hired and existing personnel
- Conducted individual clinical mentorship sessions

- Provided selected nurses with intensive hands-on clinical skills placement to expand NICU skills
Zambia

- Trained district mentorship teams who then held monthly on site health facility staff training and mentorship visits on normal delivery and partograph use, $\mathrm{EmONC}$, and $\mathrm{HBB}$

\section{Strategy 4. Improve quality of care and ensure care is evidence-based}

Approach 4.1: Implemented quality, effective interventions to prevent and treat obstetric and newborn complications

- Provided quality improvement practice to increase partograph use

- Implemented KMC

Approach 4.2: Introduced sound managerial practices using 'short-loop' data feedback and response to ensure reliable delivery of quality essential and emergency maternal and newborn care

Approach 4.3: Developed guidelines and policies, and ensured protocol adherence
- Incorporated concepts related to respectful maternity care into customer care training of midwives

- Used facility-generated data to review quality of care and implement practice changes

- Developed national standards for MDSR that were informed by SMGL processes

- Implemented BABIES matrix to prevent perinatal deaths by using data to guide actions
- Introduced emergency kits and logs/registers to facilitate quick access to emergency supplies

- Implemented partograph use by facility staff

- Enhanced infection prevention practices

- Incorporated respectful maternity care into EmONC and early newborn care and supported it through mentorship

- Developed clinical guidelines and protocols for diagnosing and managing most common obstetric emergencies

- Contributed to the development of the newborn health framework and guidelines

- Created standardized clinical forms to guide providers in recognizing danger signs and diagnosing the most common obstetric emergencies

- Introduced laminated checklists for quick reference in delivery rooms

\section{Strategy 5. Ensure referral capacity exists to support transfers to higher-level care}

Approach 5.1: Improved referral com- - Introduced ambulance referral forms to better munication systems

track referrals
- Set up and supported district ambulance committees to work on referral-related issues

- Procured and maintained landline phones for facilities and mobile phones for village health workers

Approach 5.2: Support increased transportation between facilities with motor vehicles or ambulances
- Procured ambulances (vehicle and tricycle)
- Used referral forms to improve communication between health centers and hospitals

- Set up and supported district ambulance committees to work on referral-related issues.

- Repaired and maintained 2-way radios at health facilities.

- Improved communications through the SMS and Remind-mi mHealth program (local communication programs)

- Procured ambulances (vehicle and motorcycle) 
TABLE 1. Continued

Country-Specific Interventions ${ }^{a}$

\begin{tabular}{|c|c|c|}
\hline \multirow[b]{2}{*}{ Strategies and Approaches } & \multirow[b]{2}{*}{ Uganda } & \\
\hline & & Zambia \\
\hline \multicolumn{3}{|c|}{ Strategy 6. Support effective maternal and perinatal health surveillance } \\
\hline $\begin{array}{l}\text { Approach 6.1: Strengthen maternal } \\
\text { and perinatal mortality surveillance in } \\
\text { facilities and communities }\end{array}$ & $\begin{array}{l}\text { - Trained providers on MPDSR } \\
\text { - Set up MPDSR system, including committees to } \\
\text { identify and understand maternal and newborn } \\
\text { mortality at facilities and in communities } \\
\text { - Strengthened prospective health facility surveil- } \\
\text { lance through the MOH DHIS2 } \\
\text { - Set up POMS and RAPID to understand facility } \\
\text { maternal and perinatal mortality } \\
\text { - Developed national standards for MDSR that } \\
\text { were informed by SMGL processes }\end{array}$ & $\begin{array}{l}\text { - Established MDSR including verbal autopsies } \\
\text { at facilities with a community component } \\
\text { - Conducted MDR trainings for the district } \\
\text { medical officer and health facility staff } \\
\text { - Supported MDR at facilities }\end{array}$ \\
\hline $\begin{array}{l}\text { Approach 6.2: Promote a government- } \\
\text { owned HMIS data-gathering system to } \\
\text { accurately record every birth outcome, } \\
\text { obstetric and newborn complication, } \\
\text { and treatment at facilities }\end{array}$ & $\begin{array}{l}\text { - Trained providers and implemented BABIES } \\
\text { matrix } \\
\text { - Strengthened prospective health facility surveil- } \\
\text { lance through the MOH DHIS2 } \\
\text { - Set up POMS }\end{array}$ & $\begin{array}{l}\text { - Supported national MDSR processes and } \\
\text { expansion of MDSR to SMGL districts }\end{array}$ \\
\hline
\end{tabular}

a This list is not exhaustive and activities noted may apply to more than one approach.

Abbreviations: BABIES, birth weight and age-at-death boxes for an intervention and evaluation system; BEmONC, basic emergency obstetric and newborn care; CEmONC, comprehensive emergency obstetric and newborn care; CPAP, continuous positive airway pressure; DHIS2, District Health Information System 2; eLMIS, Electronic Logistic Management Information System; EmONC, emergency obstetric and newborn care; HBB, Helping Babies Breathe; HMIS, Health Management Information System; KMC, kangaroo mother care; MDR, maternal death review; MDSR, maternal death surveillance and response; $\mathrm{MOH}$, ministry of health; MPDSR, maternal and perinatal death surveillance and response; NICU, neonatal intensive care unit; NSCU, neonatal special care units; POMS, pregnancy outcome monitoring surveillance; RAPID, Rapid Ascertainment Process for Institutional Deaths; SMGL, Saving Mothers, Giving Life; SMS, short message service.

ention and Evaluation System (BABIES) matrix. Interviews were conducted to understand the patterns of the matrix's use, its perceived value, and behavior change that resulted from its use. All interviews were conducted in English, audio recorded, and transcribed verbatim, after which common themes were identified from the transcripts.

SMGL introduced BABIES through district workshops, facility-based mentorship, and international conferences. The BABIES report card and storyboards were used to identify 'trigger' cells for the BABIES matrix (Box 2). The report card provides a listing of at least 70 indicators that can be calculated from the matrix. SMGL nested the BABIES data within the pregnancy outcome monitoring surveillance database in a multidimensional table to evaluate the third delay using indicators to assess the performance of the system.

Verbal autopsies collected in the context of baseline and endline population mortality measurement studies captured the causes and circumstances of maternal deaths in both Uganda and
Zambia. Questionnaires administered to family members about the women's experiences during the days leading up to a maternal death were used to discern both medical and nonmedical causes of death, such as sociocultural and behavioral factors. Each interview included an open narrative where respondents provided an unprompted account of events preceding the death. Narrative data excerpts were used as a case vignette to illustrate certain barriers to appropriate facility care (Box 3).

\section{Ethics}

The study protocol was reviewed and approved by the Ugandan and Zambian Ministries of Health and deemed nonresearch by the U.S. Centers for Disease Control and Prevention Human Research Protection Office of the Center for Global Health. Written informed consent was obtained for respondents in all households and among women for the census, Reproductive Age Mortality Survey study interviews, and verbal autopsies. 
BOX 1. Definitions and Descriptions of Indicators of Interest for SMGL Monitoring and Evaluation

\begin{tabular}{|c|c|}
\hline Indicator & Description \\
\hline Performance of EmONC signal functions & $\begin{array}{l}\text { Basic Services: } \\
\text { - Administer parenteral antibiotics } \\
\text { - Administer uterotonic drugs (e.g., parenteral oxytocin) } \\
\text { - Administer parenteral anticonvulsants for pre-eclampsia and eclampsia (e.g., magnesium sulfate) } \\
\text { - Manual removal of placenta } \\
\text { - Remove retained products (e.g., manual vacuum aspiration, dilation and curettage) } \\
\text { - Perform assisted vaginal delivery (e.g., vacuum extraction, forceps delivery) } \\
\text { - Perform basic neonatal resuscitation (e.g., bag and mask) } \\
\text { Comprehensive Services: } \\
\text { - Perform surgery (e.g., caesarean section) } \\
\text { - Perform blood transfusion }\end{array}$ \\
\hline Basic facility infrastructure & $\begin{array}{l}\text { - Electricity } \\
\text { - Regular water supply } \\
\text { - Functional communications systems } \\
\text { - Motorized vehicles available } \\
\text { - Services available } 24 \text { hours a day }\end{array}$ \\
\hline Promotion of protocols and guidelines & $\begin{array}{l}\text { Facilities with protocols available and displayed on the following topics: } \\
\text { - Obstetric and newborn complications } \\
\text { - Postpartum hemorrhage } \\
\text { - Active management of the third stage of labor } \\
\text { - Helping Babies Breathe or kangaroo mother care } \\
\text { - Early newborn care }\end{array}$ \\
\hline Availability of essential drugs & - Oxytocin, magnesium sulfate, gentamycin \\
\hline
\end{tabular}

Abbreviation: EmONC, emergency obstetric and newborn care.

\section{RESULTS}

\section{Strategies, Interventions, and Selected Quantitative Results}

The 6 strategies implemented through SMGL are summarized in Table 1 and described below. Outcomes resulting from the implementation of these strategies are presented in Table 2 (Uganda) and Table 3 (Zambia) with selected outcomes highlighted below.

\section{In both Uganda and Zambia, the total number of BEmONC facilities more than doubled between baseline and endline.}

\section{Strategy 1. Ensure Facilities Have Adequate Infrastructure to Provide EmONC}

Adequate infrastructure is needed to provide safe delivery care and implement EmONC functions. In both countries, SMGL sought to improve basic facility infrastructure and enhance the facilities' ability to provide safe deliveries 24 hours a day/ 7 days a week (24/7) (Table 1). In Uganda, SMGL supported renovation and upgrading of operating theatres and made facility infrastructure changes to enlarge labor rooms, to create neonatal special care units and to provide spaces for kangaroo mother care, a program that has been shown to reduce neonatal mortality by promoting early skin-to-skin contact and improving thermoregulation in low birth weight and preterm newborns. In Zambia, SMGL increased the number of delivery beds and refurbished rooms to enable increased volume of facility deliveries, longer postpartum stays, and kangaroo mother care. Both countries focused on improving facility availability of electricity and water.

In Uganda, the total number of BEmONC and CEmONC facilities more than doubled between baseline and endline (BEmONC from 3 to 8 and CEmONC from 7 to 17) (Table 2). In Zambia, the number of BEmONC facilities more than doubled (from 3 to 8 ) and CEmONC facilities increased by $25 \%$ (from 4 to 5 ) (Table 3 ). This expansion of EmONC facilities is reflected in a corresponding 


\section{Simplest BABIES Matrix}

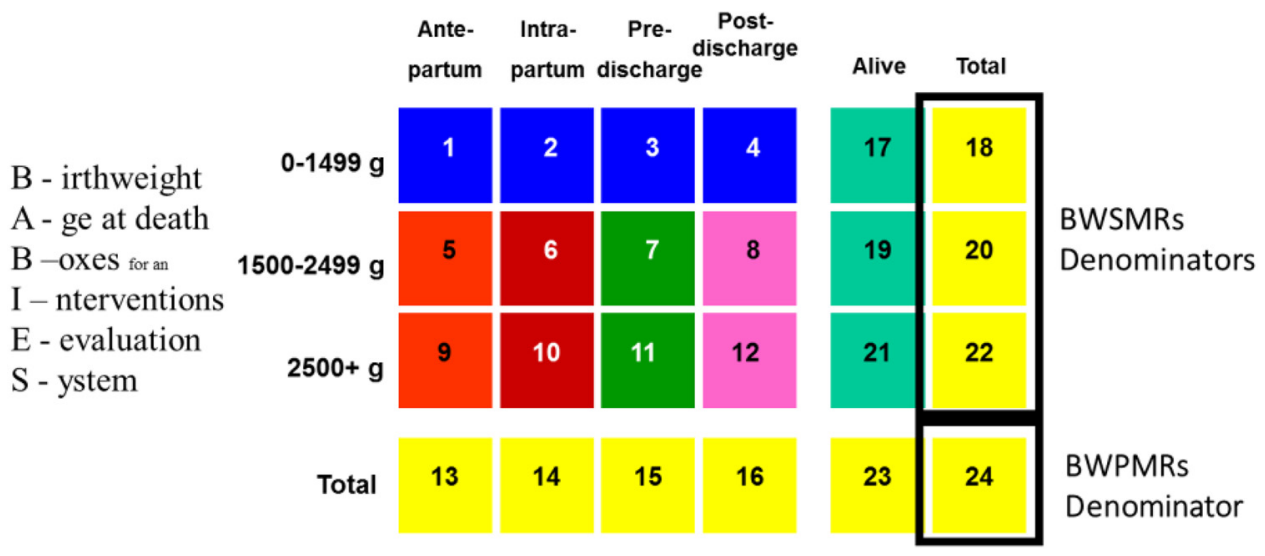

Saving Mothers, Giving Life (SMGL) used the Birth Weight and Age-at-Death Boxes for Intervention and Evaluation System (BABIES) ${ }^{35}$ matrix as a basic surveillance tool to link maternal and perinatal outcomes with evidenced-based interventions. The BABIES matrix is a simple, expandable, and adaptable table that displays stillbirths and newborn deaths by birthweight and time of death. The matrix accounts for every mother and stillbirth/newborn pair in a facility. Its indicators empower facility staff to monitor and evaluate coverage, equity, and quality-of-care indicators for their patients.

In its simplest form, completion of the BABIES matrix allows facilities to calculate 2 types of mortality indicators. First, birthweight proportionate mortality rates (BWPMRs) help to answer the question "Have we chosen the right thing to do?" Second, birthweight specific mortality rates (BWSMRs) help to answer the question "Are we doing these things right?"

statistically significant increase in the proportion of deliveries occurring in EmONC facilities between baseline and endline for both countries (Uganda from $28.2 \%$ to $41.0 \%$; Zambia from $26.0 \%$ to $29.1 \%$ ). The availability of facility electricity increased significantly in both countries (by 69\% in Uganda and $67 \%$ in Zambia), and water availability in facilities improved (Uganda from 76.2\% to $100 \%$; Zambia from $90.0 \%$ to $97.3 \%$ ).

\section{Strategy 2. Ensure Sufficient Medical Supplies, Equipment, and Medications}

In Uganda, SMGL procured essential equipment, including surgical equipment, EmONC supplies, and commodities; strengthened supply chains for essential medicines; upgraded BEmONC facilities; and strengthened the blood supply system in CEmONC sites (Table 1). In Zambia, SMGL supported the procurement of essential medications and implemented logistics management systems to reduce or eliminate stock-outs or supply depletion. Specialized equipment for surgeries, treatment of postpartum hemorrhage, and neonatal resuscitation were obtained for higher-level care along with additional supplies for BEmONC sites. Project C.U.R.E supplied donated facilityspecific, essential equipment and commodities shipping 16 containers to Uganda and 20 to Zambia over the life of the initiative.

The proportion of facilities with no stock-outs of oxytocin significantly increased between baseline and endline in Uganda, from 56.2\% to $81.9 \%$, but did not change in Zambia. The proportion of facilities with no stock-outs of magnesium sulfate significantly increased in both countries: Uganda increased from $46.7 \%$ to $63.8 \%$ and Zambia increased from $20.0 \%$ to $43.0 \%$. In some cases, however, the availability of essential drugs was unchanged or even declined between baseline and endline. For example, the current availability of gentamicin in Zambia decreased from $67.3 \%$ to $48.2 \%$, but remained stable in Uganda 
BOX 3. Verbal Autopsy Case Example From SMGL District Verbal Autopsy Case Example of the Third Delay: Sarah's Story

Sarah was a 17-year-old from Uganda who died after giving birth to her first child. The respondent during the verbal autopsy interview was Sarah's mother-in-law. The interview was transcribed and summarized below. The names of persons and places and the dates have been changed to protect confidentiality.

Labor pains started on October 14, 2016 at $8 \mathrm{am}$. Sarah [the pregnant woman] noted some blood coming from her vagina. She told me what was happening, and I told her to go the hospital if the situation worsened. Instead, Sarah went to work in the garden since the pains were not strong. Early in the morning on the following day, the labor pains increased, and Sarah's husband took us to the hospital on a motorcycle, a 25-minute drive away. Upon reaching the labor ward, Sarah was attended to immediately and soon gave birth. However, shortly after giving birth, she started bleeding heavily, and the midwife started 'scooping' blood from the bed and gave Sarah an injection to stop the bleeding. The midwife called the doctor who came immediately. The doctor took Sarah to the operating theatre to establish the cause of the bleeding and told me to go and buy two sachets of blood. I rushed to the health center where I found only one sachet remaining. By the time I purchased the sachet and Sarah was transfused, it was almost $4 \mathrm{pm}$. I checked on Sarah and found blood coming from her nose; she was unconscious. The nurse told me that they would refer her to a higher level hospital, but I said no, seeing my daughter-in-law in a passing (unconscious) state. Just before $5 \mathrm{pm}$, right after I completed that statement, Sarah died. The baby remained alive. According to my feeling, the death could not be avoided; it was her time to die-the day that God had planned.

In both Uganda
and Zambia,
SMGL supported
recruitment of
health care staff in
order to meet
national
standards for
CEmONC facilities.

at $90.5 \%$ at baseline and $88.6 \%$ at endline (Table 2 and Table 3).

\section{Strategy 3. Ensure Sufficient Trained Health Care Providers at Facilities}

In both Uganda and Zambia, SMGL supported recruitment of new providers for facilities in the SMGL districts (Table 1). In Uganda, the facilities hired doctors and nurse midwives at sufficient numbers to meet national standards for newly opened CEmONC facilities, and in Zambia, staffing increases focused on midwives. In Uganda, district leadership elected to maintain midwives and EmONC-trained nurses within the maternity ward rather than rotating staff, which was typical in most facilities. SMGL supported staff training of anesthetists, doctors, midwives, and nurses to expand their skills in the provision of EmONC services (Table 1). In Uganda and Zambia, providers received training in EmONC; clinical decision making; treatment of obstetric complications, including obstetric hemorrhage and eclampsia; surgical skills; and neonatal resuscitation using the Helping Babies Breathe curriculum. In Uganda, clinical officers received surgical skills training on medical decision making and cesarean section. In Zambia, midwives were trained to manage hemorrhages, including using a uterine balloon tamponade to treat postpartum hemorrhage. In both countries, mentorship played a key role in long-term staff development and support, especially for midwives. In Uganda, mentorship included intensive mentoring of obstetricians and individual follow-up visits with mentees. ${ }^{36}$ In Zambia, SMGL implemented a training-oftrainers model where district mentorship teams were trained to mentor others followed by monthly on site training that included drills and clinical mentorship visits.

In Uganda, the increase in 24/7 care from $74.6 \%$ to $82.9 \%$ was not significant (Table 2 ) but, in Zambia, staffing increases contributed to a higher proportion of health centers providing 24/7 care at endline compared with baseline (from $64.8 \%$ to $95.5 \%$ ) (Table 3). Facilities in Uganda reported improvements in the performance of several EmONC signal functions in the 3 months prior to the HFAs, including administration of parenteral oxytocin (from $69.5 \%$ to $98.1 \%$ ), manual removal of placenta (from $28.6 \%$ to $54.3 \%$ ), removal of retained products (from $19.0 \%$ to $61.9 \%$ ), and newborn resuscitation (from $34.3 \%$ to $87.6 \%$ ) (Table 2). There was either a nonsignificant change or reduction in the proportion of facilities performing the remaining signal functions at endline compared with baseline. In Zambia, a significant increase in the proportion of facilities performing 2 signal functions was observed-for removal of retained products (from $17.3 \%$ to $49.1 \%$ ) and newborn resuscitation (from $27.3 \%$ to $74.6 \%$ ) with no significant change in other signal functions (Table 3).

\section{Strategy 4. Improve Quality of Care and Ensure Care is Evidence-Based}

Overall, SMGL focused on providing tools to help strengthen provider practices, developing guidelines and policies, and ensuring protocol adherence (Table 1). In Uganda, the project implemented kangaroo mother care in SMGL facilities and trained providers on simplified basic resuscitation, using the Helping Babies Breathe 
TABLE 2. Monitoring and Evaluation Outcomes Associated With Strategies to Reduce the Third Delay in Uganda, 2011-2016 ( $N=105$ facilities)

\begin{tabular}{|c|c|c|c|c|}
\hline Indicators & $\begin{array}{l}\text { Baseline }^{a} \\
\text { Value }\end{array}$ & $\begin{array}{l}\text { Endline }^{a} \\
\text { Value }\end{array}$ & $\%$ Relative Change ${ }^{b}$ & Sig. Level ${ }^{c}$ \\
\hline \multicolumn{5}{|l|}{ Strategy 1: Ensure facilities have adequate infrastructure to provide EmONC } \\
\hline Total number of EMONC facilities & 10 & 25 & 150.0 & $\mathrm{~N} / \mathrm{A}$ \\
\hline Number of CEmONC facilities & 7 & 17 & 142.9 & N/A \\
\hline Number of BEmONC facilities & 3 & 8 & 166.7 & N/A \\
\hline Deliveries in EmONC facilities & $28.2 \%$ & $41.0 \%$ & 45.4 & $* * *$ \\
\hline Hospitals/health center IVs that perform blood transfusions ${ }^{d}$ & $56.3 \%$ & $100.0 \%$ & 77.6 & N/A \\
\hline $\begin{array}{l}\text { Hospitals/health center IVs that have capacity to perform surgery } \\
\text { (caesarean-section) }^{d}\end{array}$ & $50.0 \%$ & $100.0 \%$ & 100.0 & $\mathrm{~N} / \mathrm{A}$ \\
\hline Facilities with electricity & $57.1 \%$ & $96.2 \%$ & 68.5 & $* * *$ \\
\hline Facilities with water & $76.2 \%$ & $100.0 \%$ & 31.2 & N/A \\
\hline \multicolumn{5}{|l|}{ Strategy 2: Ensure sufficient medical supplies and medications } \\
\hline Facilities experiencing no stock-out of oxytocin in the past 12 months & $56.2 \%$ & $81.9 \%$ & 45.7 & *** \\
\hline Facilities experiencing no stock-out of magnesium sulfate in the past 12 months & $47.6 \%$ & $63.8 \%$ & 34.0 & *** \\
\hline Facilities reporting gentamycin antibiotic currently available & $90.5 \%$ & $88.6 \%$ & -2.1 & NS \\
\hline \multicolumn{5}{|l|}{ Strategy 3: Ensure sufficient trained health care providers at facilities } \\
\hline Facilities reporting at least 1 doctor, nurse, or midwife is on staff & $100.0 \%$ & $100.0 \%$ & 0.0 & NS \\
\hline Health center Ills that are open $24 / 7^{e}$ & $74.6 \%$ & $82.9 \%$ & 11.1 & NS \\
\hline \multicolumn{5}{|l|}{ Facilities reporting EmONC lifesaving interventions performed in the past 3 months ${ }^{f}$} \\
\hline Parenteral antibiotics & $85.7 \%$ & $92.4 \%$ & 7.8 & NS \\
\hline Parenteral oxytocin & $69.5 \%$ & $98.1 \%$ & 41.2 & *** \\
\hline Parenteral anticonvulsants & $48.6 \%$ & $34.3 \%$ & -29.4 & ** \\
\hline Manual removal of placenta & $28.6 \%$ & $54.3 \%$ & 89.9 & *** \\
\hline Remove retained products & $19.0 \%$ & $61.9 \%$ & 225.8 & $* * *$ \\
\hline Assisted vaginal delivery & $4.8 \%$ & $10.5 \%$ & 118.8 & NS \\
\hline Newborn resuscitation & $34.3 \%$ & $87.6 \%$ & 155.4 & $* * *$ \\
\hline
\end{tabular}

Strategy 4: Improve quality of care and ensure care is evidence-based

Facilities with protocols and guidelines available and displayed on EmONC lifesaving interventions

\begin{tabular}{|c|c|c|c|c|}
\hline AMTSL & $39.0 \%$ & $58.1 \%$ & 49.0 & *** \\
\hline Eclampsia or magnesium sulfate use & $8.6 \%$ & $74.3 \%$ & 764.0 & *** \\
\hline Immediate newborn care & $30.5 \%$ & $79.0 \%$ & 159.0 & *** \\
\hline Facilities that report routine practice of partograph & $33.3 \%$ & $92.4 \%$ & 177.5 & *** \\
\hline Facilities reporting that obstetric patients never share beds & $35.2 \%$ & $91.4 \%$ & 159.7 & *** \\
\hline Facilities reporting that women never deliver on the floor & $85.7 \%$ & $91.4 \%$ & 6.7 & NS \\
\hline
\end{tabular}


TABLE 2. Continued

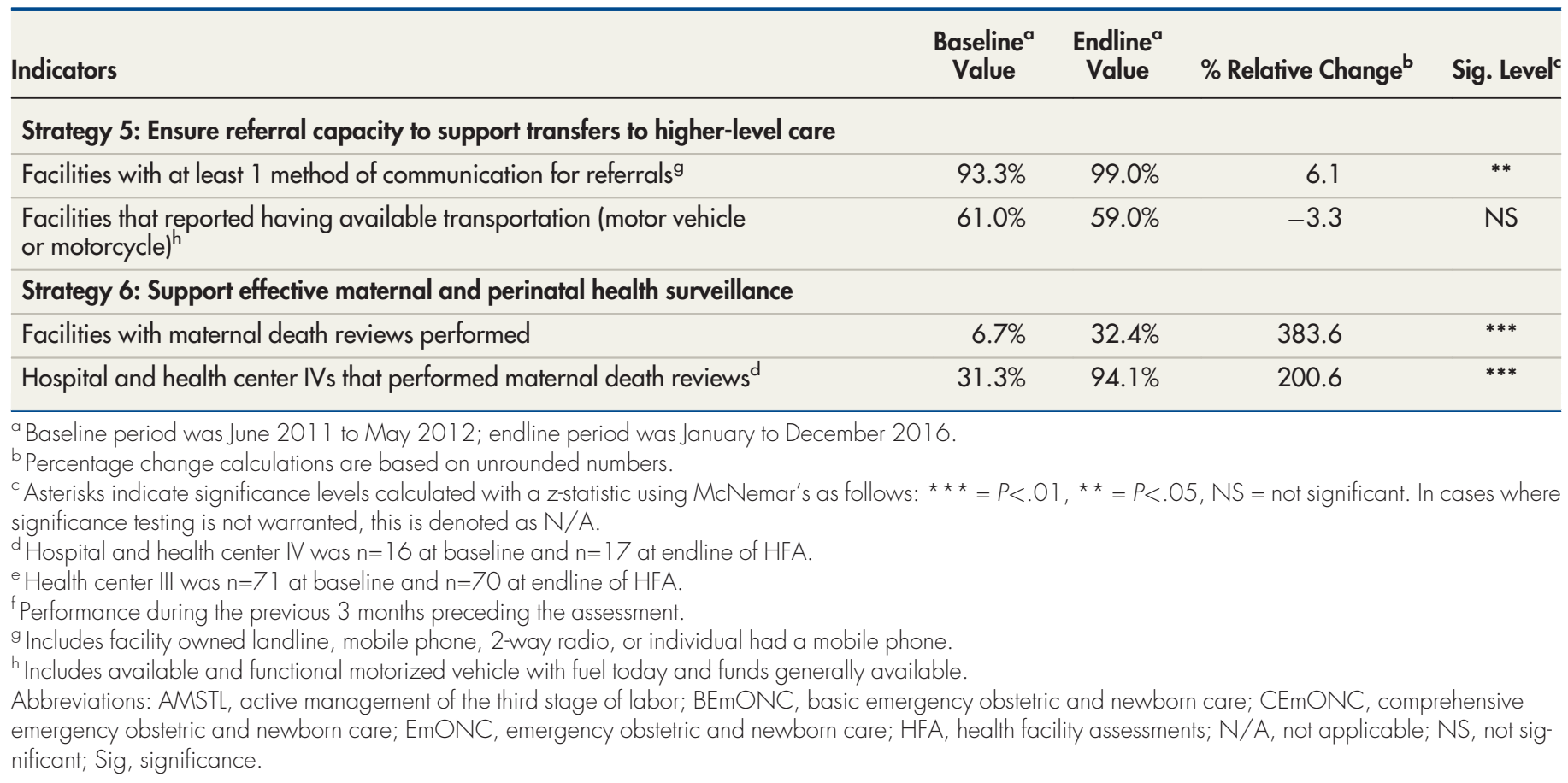

curriculum, ${ }^{37}$ and categorizing infant outcomes by birthweight and age at delivery, using the BABIES clinical tool. ${ }^{38}$ In Zambia, guidelines, protocols, and mini-emergency kits were developed to manage common EmONC emergencies, clinical forms were standardized to guide providers to recognize obstetric danger signs, and checklists were introduced for reference in delivery rooms. Respectful maternity care concepts were introduced into midwife trainings in both countries. Additionally, in both countries, maternal death surveillance and response guidelines were developed and implemented to systematize and strengthen maternal death reviews.

In Uganda, the proportion of facilities with

In Uganda and Zambia, referral forms were introduced, ambulances were procured to assist in transport to and between facilities, and district ambulance committees were formed to strengthen referrals. increased significantly between baseline and endline (from $33.3 \%$ to $92.4 \%$ ), whereas data were not available in Zambia at both time periods. Partograph use was visually verified by surveyor. The proportion of facilities reporting routine practice of active management of third stage of labor increased significantly in both Uganda (from $75.2 \%$ to $96.2 \%$ ) and Zambia (from $71.8 \%$ to $95.5 \%)$. The proportion of facilities that reported women never shared beds increased significantly in Uganda (from $35.2 \%$ to $91.4 \%$ ), but not in Zambia (from $62.7 \%$ to $73.6 \%$ ) (Table 2 and Table 3).

\section{Strategy 5. Ensure Referral Capacity to Support Transfers to Higher-Level Care}

SMGL supported improved referral communication systems and invested in improving transportation between facilities by acquiring additional motor vehicles or ambulances (Table 1). In Uganda and Zambia, referral forms were introduced, ambulances-motor vehicles, motor bikes/tricycles-were procured to assist transportation to and between facilities, and district ambulance committees were formed to strengthen referrals. 
TABLE 3. Monitoring and Evaluation Outcomes Associated With Strategies to Reduce the Third Delay in Zambia, 201 1-2016 ( $N=110$ facilities)

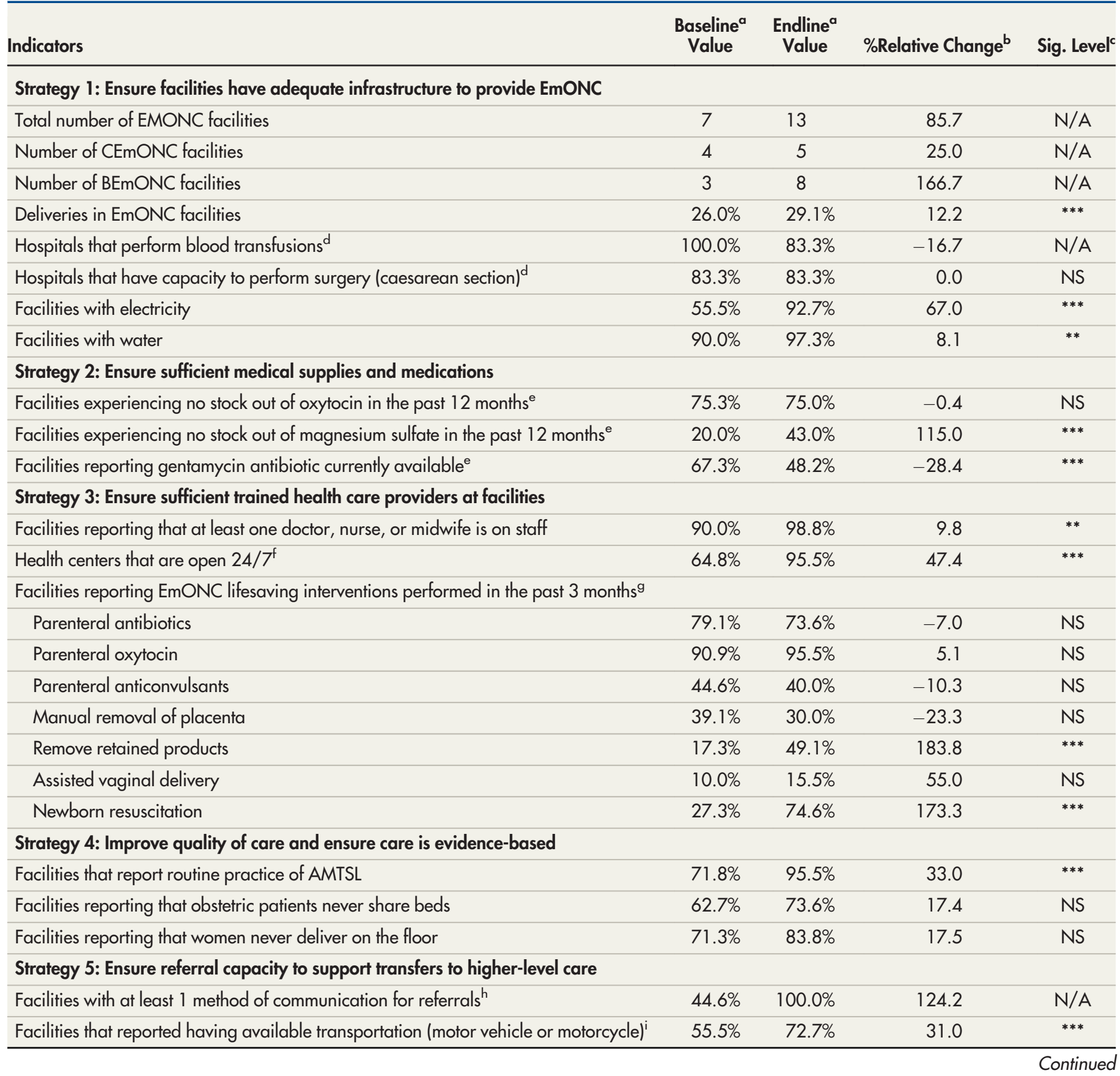


TABLE 3. Continued

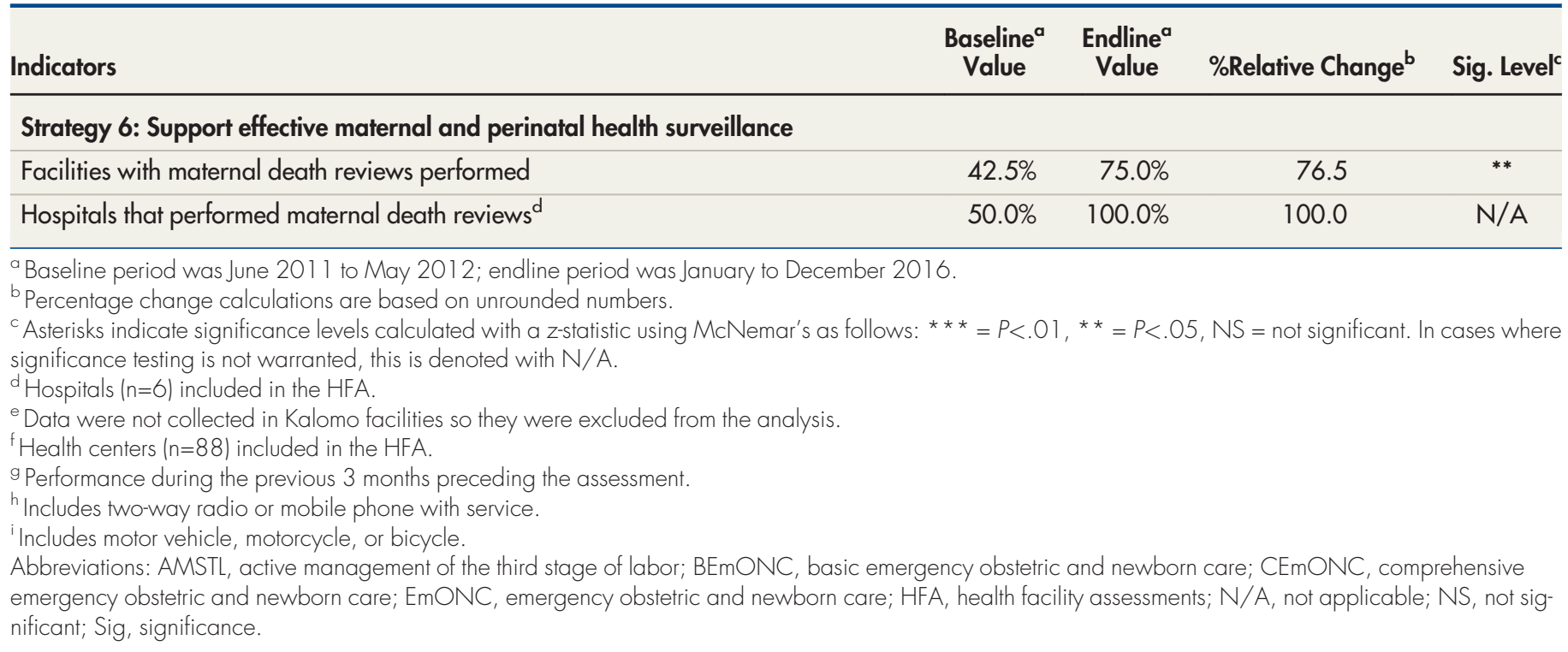

In Uganda, the data showed a significant increase in the proportion of facilities that reported at least 1 method of communication (e.g., telephones and radios) for referral (from 93.3\% to $99.0 \%$ ), but there was no noted improvement in transport availability (from $61.0 \%$ to $59.0 \%$ ) (Table 2). In Zambia, 100\% reported having at least 1 method of communication at endline (up from $44.6 \%$ at baseline), and the proportion of facilities with available transportation significantly increased (from $55.5 \%$ to $72.7 \%$ ) (Table 3 ).

\section{Strategy 6. Support Effective Maternal and Perinatal Health Surveillance}

In both countries, SMGL strengthened maternal and perinatal mortality surveillance in facilities and communities and supported governmentmanaged data processes (Table 1). In Uganda, SMGL helped establish maternal and perinatal death surveillance and response committees, enhanced facility surveillance processes and sys-

The daily visibility of the matrix in the ward was important for increasing awareness and effectiveness. tems to capture more refined health facility and outcome data, and strengthened the national health management information system. In Zambia, SMGL established the maternal death surveillance and response, including the use of verbal autopsies at facilities and in communities; maternal death review trainings for district and health facility staff; and implementation of death reviews.
Significant increases in the proportion of facilities conducting maternal death reviews were observed in Uganda (from $6.7 \%$ to $32.4 \%$ ) and Zambia (from $42.5 \%$ to $75.0 \%$ ). In Uganda, a significantly higher proportion of hospitals and health center IVs reported performing maternal death reviews at endline compared with baseline (from $31.3 \%$ to $94.1 \%$ ). Similarly, in Zambia, $100 \%$ of hospitals reported performing maternal death reviews at endline (up from $50.0 \%$ at baseline) (Table 2 and Table 3).

\section{Qualitative Results}

The BABIES matrix, implemented in select facilities in Uganda, provided a simple, systematic approach to monitor and evaluate staffing coverage, equity, and quality of care for facility service populations (Box 2).

The aim of the 16 in-depth interviews conducted in November 2017 in Uganda was to better understand the patterns of BABIES matrix use, its perceived value, and behavior change that resulted from its use. Providers described use of the BABIES matrix on a primarily monthly basis during maternity and all-staff meetings and perceived that the matrix was highly valuable, with nearly all providers reporting that it instilled a strong sense of accountability for perinatal deaths that simply had not existed previously. One doctor said, "the first time we ever projected the information, it was an eye-opener. . . . Oh! It's just not 
numbers, it's real figures that can influence outcome." The daily visibility of the matrix in the ward to providers, clients, and visitors alike played an important role in increasing its effectiveness as a tool for awareness.

Providers shared numerous examples of how the BABIES matrix has led to positive changes in the facility, including improved labor monitoring and management, use of the partograph, more honest intercadre communication, better communication and outreach with lower-level facilities, and more complete and accurate documentation, all of which they believe contributed to improved quality of care. When asked if the BABIES matrix will continue to be used after SMGL ends, nearly all providers reported they believed it would. One midwife said, "Yes, whether the SMGL continues, or whether it stops, the BABIES matrix board has to continue. Because whatever we are doing, we are not doing it for SMGL, we are doing it for the better management of our mothers and babies!"

Verbal autopsy narratives give context to the facility results by providing descriptions of women's experiences prior to a maternal death. The vignette in Box 3 highlights a woman's experience seeking care and the delays she encountered. This example emphasizes the importance of blood and referrals to facilities that can provide CEmONC.

\section{DISCUSSION}

Successful interventions to reduce maternal and perinatal mortality should ensure that women deliver in facilities with the capabilities and staff to manage both expected and emergent complications. The World Health Organization strongly advocates for all births to be assisted by skilled attendants. ${ }^{39}$ Women are encouraged to give birth in health care facilities to ensure access to skilled health care professionals and timely referral to higher-level facilities for management of obstetric complications, if they occur. However, increasing facility-based delivery rates and EmONC capabilities alone will not ensure that the full range of barriers to appropriate care are addressed. Quality of care depends on a host of factors that SMGL only partially measured. Timeliness and appropriateness of referrals, accuracy of provider decision making and diagnoses, and quality of care provided are only a few factors that contribute to reducing deaths due to the third delay.

\section{SMGL's Successes}

SMGL implemented 6 strategies to reduce deaths due to the third delay by providing the most timely and appropriate delivery care for women and their newborns. In SMGL districts in Uganda and Zambia, the number of CEmONC and BEmONC facilities increased, offering women greater access to maternity services. SMGL enhanced facility capabilities by providing additional infrastructure support and developed processes for medication and supply stocking. SMGL supported increased staffing, trained and mentored staff to implement evidence-based interventions, and provided communication and transportation systems for maternal transfers. SMGL's investments in the availability and accessibility of maternity health services were crucial to increasing the proportion of facility deliveries observed in both Uganda and Zambia and to decreasing maternal and perinatal deaths. ${ }^{32,40}$

During Phase 2, the SMGL initiative focused on both the mother and newborn. Historically, many EmONC programs have focused heavily on either maternal or newborn health care rather than the provision of effective care for both women and newborns. ${ }^{41-43}$ Investing in facilitybased care for both the mother and newborn, using available interventions, has been found in one study to avert an estimated $71 \%$ of newborn deaths, $33 \%$ of still-births, and $54 \%$ of maternal deaths. ${ }^{44}$ Along with the noted extensive investments in maternal health, SMGL allocated substantial resources dedicated to perinatal survival-improved equipment and expanded training of providers, building of surveillance infrastructure for perinatal mortality, and improved data to action through BABIES. This expanded capacity is likely to have contributed to the overall reduction in perinatal mortality.

SMGL improved data systems, including health outcome information for the monitoring and evaluation of facility performance and accelerated implementation of death reviews to better understand remaining gaps in care and prevent future deaths. The initiative also supported national initiatives and expanded surveillance systems to improve the quality and specificity of maternal and perinatal data for action through maternal death surveillance and response systems. Improved identification, notification, and determination of causes and preventability of maternal deaths allowed decision makers and providers to develop and implement targeted improvements. Expansion of the maternal death surveillance and response system to include perinatal death identification and reviews and implementation of the BABIES matrix in Uganda provided guidance for how to improve newborn
"[W]hether the SMGL continues, or whether it stops, the BABIES matrix board has to continue. Because whatever we are doing, we are not doing it for SMGL, we are doing it for the better management of our mothers and babies!" 
The SMGL endline evaluation found that the maternal mortality ratio in facilities was still unacceptably high in both Uganda and Zambia.

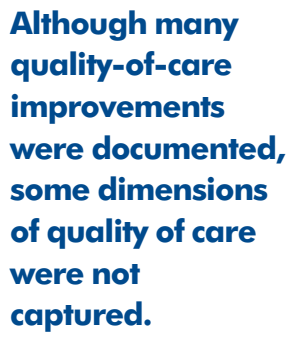

health. These enhanced surveillance systems, built on existing data infrastructure and supported by development of national guidelines, are a lasting contribution to national data systems for tracking maternal and perinatal mortality over time.

\section{Limitations of the SMGL Approach and Monitoring and Evaluation Methods}

Despite the notable achievements of the SMGL initiative including a documented reduction in facility maternal mortality ratio of $43.8 \%$ in Uganda and $37.6 \%$ in Zambia, the SMGL endline evaluation found that the maternal mortality ratio in facilities was still unacceptably high: 300 maternal deaths per 100,000 live births in Uganda and 231 maternal deaths per 100,000 live births in Zambia. In both countries, although the majority of women delivered in health facilities $(66.8 \%$ in Uganda; $90.2 \%$ in Zambia), most of the maternal deaths occurred in facilities, ${ }^{1}$ a clear indication that critical gaps remain in improving care and preventing maternal deaths after women reach a health care facility.

More comprehensive studies are needed to document the impact of BABIES on perinatal survival and identify remaining gaps. SMGL efforts demonstrated a reduction in institutional perinatal mortality rates and stillbirth rates in both countries; however, no significant change was found in predischarge neonatal mortality rates. Additional work is also needed to better characterize contributors and interventions needed to impact early neonatal mortality.

SMGL improved facility infrastructure, equipment, and supplies during the initiative. However, the slow and uneven pace of the upgrades was a concern raised during the initiative. ${ }^{36}$ Some sites reported additional needs for refurbishment that were not accomplished, such as further expanding CEmONC capacity, particularly in Zambia. ${ }^{36}$ Additionally, with increased demand for services, the need for essential medications also increased. However, sites noted that the increased supply was not necessarily matched to their need. ${ }^{36}$ Infrastructure and supply chain barriers may have hampered the impact of the initiative on addressing the third delay. The challenges related to these systems are not unique for health programs in resource limited settings, further investment is needed to support these systems in order to achieve real impact.

Although extensive monitoring and evaluation activities were implemented as part of the
SMGL initiative, the methods still had important limitations. In Zambia, at baseline, the implementing partners developed unique tools and systems for facility data collection that were not harmonized across districts, and some indicators could not be aggregated at baseline. As census and verbal autopsy data are dependent on secondary reporting by household informants, recall bias may have affected the reported timing and determination of live births, deaths, and health history provided for the deceased. Additionally, the interventions were not evaluated independently, making it impossible to determine the relative impact of any individual intervention.

\section{Quality of Care is Essential}

To address the third delay, identifying barriers in facility-based service provision is critical. In the case vignette of a woman's experience seeking care and the delays she encountered, Sarah's mother-in-law described the facility care and multiple barriers she encountered: lack of active interventions to prevent hemorrhage in the immediate postpartum period, lack of urgent blood transfusion protocol, insufficient blood supply, and a delay in referral to a facility that could provide CEmONC. At each step of her care, these delays compounded Sarah's critical health status. Understandably, stories like these influence other women's decisions about whether to deliver in a facility. ${ }^{21}$

SMGL documented numerous quality-of-care improvements, including increased availability and use of evidence-based protocols and practices. However, SMGL monitoring and evaluation did not capture some dimensions of quality of care, including important areas such as intrapartum monitoring, time from admission or decision to surgery for cesarean deliveries, adequacy of neonatal resuscitation, and effectiveness of care for small and sick babies. Respectful care at birth is an emerging area that SMGL did not measure. Models of quality-of-care assessment that include observational and structured interviews to allow for more refined measurement of maternity care processes and outcomes do exist and would have been a valuable addition to the routine monitoring implemented by SMGL. ${ }^{45}$

\section{Ensuring Sustainability}

Additional resources will be required to maintain the advances that the SMGL initiative achieved in addressing barriers that contribute to third delay related maternal deaths. Strengthening and 
expanding existing national systems for service delivery-including maternal and newborn health, HIV, family planning, and immunization services-are essential to achieve future gains.

The establishment of sufficient human resources for health requires countries to align national and local policies and programs to ensure equitable access to health workers. ${ }^{46}$ In Uganda, the SMGL initiative aligned wages to national standards, transferred human resource management to the national systems to improve sustainability, and limited rotation of specialized staff away from maternities. Expanding preservice training may provide staff with the crucial skills needed sooner, rather than relying on and waiting for in-service training. Tailoring effective interventions to each site would help establish sufficient health care providers for the future. If supported by the national system, task shiftingwhere tasks are moved to less specialized health workers-can help improve health system efficiency, expand coverage, and save costs. ${ }^{47}$ Other strategies that could be implemented include quality competitions and performance-based financing. ${ }^{48,49}$

Although SMGL provided extensive health care provider training, the first year evaluation found only a modest increase (10\%) in providers' obstetric knowledge. ${ }^{50}$ After the interim assessment, the initiative incorporated active learning, which has been demonstrated to be effective for health workers, ${ }^{51}$ into their skill-building activities; this included interactive obstetric drills and ongoing mentorship, emphasizing the importance of ongoing reinforcement of skills. Further assessment of gaps in knowledge and practice would be useful to tailor trainings to ensure providers maintain and strengthen skills. Linking certification and accreditation with continuing medical education has been implemented in some settings to maintain skills, and models have been developed for low-resource settings. ${ }^{52}$ Investment in ongoing training and sustained mentorship and coaching will help staff retain skills and strengthen clinical practice. ${ }^{53}$ Sites should explore lowerresource intensive models of mentorship to ensure that providers are supported and practices are evidence-based. ${ }^{54,55}$ Institutionalizing systems for monitoring and retraining staff in nationallevel policies can help ensure appropriate standards of care. To address that, SMGL enhanced surveillance and strengthened national data collection systems and trained hundreds of facility personnel in improved data capture methods. Further support of these systems is essential to continue to provide improved data for decision making and performance evaluation. Integrating disease surveillance within the national health information system can create a valuable data resource that can inform changes in not only maternal and perinatal mortality but also noncommunicable diseases and emerging epidemics.

\section{CONCLUSION}

The SMGL initiative focused on reducing maternal and perinatal mortality during the critical period around labor, delivery, and immediately postpartum. SMGL implemented 6 comprehensive intervention strategies to focus on the third delay: (1) improving infrastructure to provide EmONC, (2) ensuring sufficient supplies, equipment, and medications, (3) ensuring sufficient trained health care providers at facilities who (4) practice quality evidence-based clinical care, (5) supporting referrals to allow transfers to higher-level care, and (6) supporting effective maternal and perinatal surveillance systems. Implementation of these key strategies was associated with significant reductions in facility maternal and perinatal mortality in Uganda and Zambia over the 5-year SMGL initiative. Further improvements are needed, as maternal and perinatal mortality levels are still unacceptably high. Stakeholders need to leverage the gains made by and sustain the momentum of SMGL and continue efforts to ensure no mother or newborn dies a preventable death.

Acknowledgments: The authors want to thank the country teams, partners, and women and families in SMGL districts in Uganda and Zambia who supported and participated in the initiative. The authors would also like to thank Yasamin Zia, who conducted the literature review and ensured references were accurate.

Funding: Saving Mothers, Giving Life implementation was primarily funded by the Office of the Global AIDS Coordinator, the U.S. Agency for International Development (USAID), Washington, D.C., the Centers for Disease Control and Prevention (CDC), Atlanta, Georgia (Cooperative agreements GPS003057 and GPS002918), Merck for Mothers, and Every Mother Counts. The funding agencies had no influence or control over the content of this article.

Disclaimer: The authors' views expressed in this publication do not necessarily reflect the views of the U.S. Government.

Competing Interests: None declared.

\section{REFERENCES}

1. Conlon CM, Serbanescu F, Marum L, et al. Saving Mothers, Giving Life Working Group. Saving Mothers, Giving Life: it takes a system to save a mother. Glob Health Sci Pract. 2019;7(suppl 1):S6-S26. CrossRef

2. Thaddeus $S$, Maine D. Too far to walk: maternal mortality in context. Soc Sci Med. 1994;38(8):1091-1110. Medline

\section{Integrating \\ disease \\ surveillance \\ within the \\ national health \\ information \\ system can create \\ a valuable data \\ resource that \\ could inform \\ changes in not \\ only maternal and \\ perinatal \\ mortality but also \\ noncommunicable \\ diseases and \\ emerging \\ epidemics.}


3. Say L, Chou D, Gemmill A, et al. Global causes of maternal death: a WHO systematic analysis. Lancet Glob Health. 2014;2(6):e323e333. CrossRef. Medline

4. Liu L, Oza S, Hogan D, et al. Global, regional, and national causes of under-5 mortality in 2000-15: an updated systematic analysis with implications for the Sustainable Development Goals. Lancet. 2016;388(10063):3027-3035. CrossRef. Medline

5. United Nations Children's Fund (UNICEF). Committing to Child Survival: A Promise Renewed Progress Report 2014. New York: UNICEF; 2014. https://www.unicef.org/publications/index 75736. html. Accessed October 12, 2018.

6. Paxton A, Maine D, Freedman L, Fry D, Lobis S. The evidence for emergency obstetric care. Int J Gynaecol Obstet. 2005;88(2):181193. CrossRef. Medline

7. Knight HE, Self A, Kennedy SH. Why are women dying when they reach hospital on time? A systematic review of the 'third delay.' PLoS One. 2013;8(5):e63846. CrossRef. Medline

8. Kyei-Nimakoh M, Carolan-Olah M, McCann TV. Access barriers to obstetric care at health facilities in sub-Saharan Africa-a systematic review. Syst Rev. 2017;6(1):110. CrossRef. Medline

9. Cannoodt L, Mock C, Bucagu M. Identifying barriers to emergency care services. Int J Health Plann Manage. 2012;27(2):e104-e120. CrossRef. Medline

10. World Health Organization (WHO), United Nations Population Fund, United Nations Children's Fund, Averting Maternal Death and Disability Program. Monitoring Emergency Obstetric Care: A Handbook. Geneva: WHO; 2009. http://www.who.int/ reproductivehealth/publications/monitoring/9789241547734/ en/. Accessed October 16, 2018.

11. Campbell OM, Graham WJ. Measuring Maternal Mortality and Morbidity: Levels and Trends. Washington, DC: The World Bank, Population and Human Resources Department, Population Health, and Nutrition Division; 1991.

12. Lawn JE, Blencowe $\mathrm{H}, \mathrm{Oza}$ S, et al.; Lancet Every Newborn Study Group. Every Newborn: progress, priorities, and potential beyond survival. Lancet. 2014;384(9938):189-205. CrossRef. Medline

13. World Health Organization (WHO). The World Health Report 2005 - Make Every Mother and Child Count. Geneva: WHO, 2005. http://www.who.int/whr/2005/en/. Accessed October 16, 2018.

14. Gebrehiwot T, San Sebastian M, Edin K, Goicolea I. Health workers' perceptions of facilitators of and barriers to institutional delivery in Tigray, Northern Ethiopia. BMC Pregnancy Childbirth. 2014;14:137. CrossRef. Medline

15. Kumar S, Dansereau E. Supply-side barriers to maternity-care in India: a facility-based analysis. PLoS One. 2014;9(8):e103927. CrossRef. Medline

16. Wilunda C, Oyerinde K, Putoto G, et al. Availability, utilisation and quality of maternal and neonatal health care services in Karamoja region, Uganda: a health facility-based survey. Reprod Health. 2015;12:30. CrossRef. Medline

17. Cham $M$, Sundby J, Vangen S. Maternal mortality in the rural Gambia, a qualitative study on access to emergency obstetric care. Reprod Health. 2005;2(1):3. CrossRef. Medline

18. Binder $P$, Johnsdotter $S$, Essén $B$. Conceptualising the prevention of adverse obstetric outcomes among immigrants using the 'three delays' framework in a high-income context. Soc Sci Med. 2012;75 (11):2028-2036. CrossRef. Medline

19. Essén B, Johnsdotter S, Binder P. Not too far to walk but too far for reciprocity: maternal mortality in a migration context using the 'three delays' framework. Int J Gynaecol Obstet. 2012;119:S343. CrossRef
20. Barnes-Josiah D, Myntti C, Augustin A. The "three delays" as a framework for examining maternal mortality in Haiti. Soc Sci Med. 1998;46(8):981-993. Medline

21. Bohren MA, Hunter EC, Munthe-Kaas HM, Souza JP, Vogel JP, Gulmezoglu AM. Facilitators and barriers to facility-based delivery in low- and middle-income countries: a qualitative evidence synthesis. Reprod Health. 2014;11(1):71. CrossRef. Medline

22. Shakibazadeh E, Namadian M, Bohren MA, et al. Respectful care during childbirth in health facilities globally: a qualitative evidence synthesis. BJOG. 2018:125(8)932-942. CrossRef. Medline

23. Kumar A, Agrawal N. Brought in dead: an avoidable delay in maternal deaths. J Obstet Gynaecol India. 2016;66(suppl 1):60-66. CrossRef. Medline

24. Combs Thorsen V, Sundby J, Malata A. Piecing together the maternal death puzzle through narratives: the three delays model revisited. PLoS One. 2012;7(12):e52090. CrossRef. Medline

25. Lori JR, Starke AE. A critical analysis of maternal morbidity and mortality in Liberia, West Africa. Midwifery. 2012;28(1):67-72. CrossRef. Medline

26. Rahman S, Choudhury AA, Khanam R, et al. Effect of a package of integrated demand-and supply-side interventions on facility delivery rates in rural Bangladesh: implications for large-scale programs. PLoS One. 2017;12(10):e0186182. CrossRef. Medline

27. Amudhan S, Mani K, Rai SK, S Pandav C, Krishnan A. Effectiveness of demand and supply side interventions in promoting institutional deliveries - a quasi-experimental trial from rural north India. Int J Epidemiol. 2013;42(3):769-780. CrossRef. Medline

28. Uganda Bureau of Statistics (UBOS), ICF International. Uganda Demographic and Health Survey 2011: Preliminary Report. Kampala, Uganda, and Calverton, MD: Uganda Bureau of Statistics and ICF International; 2012. https://www.ubos.org/onlinefiles/ uploads/ubos/UDHS/UDHS201 1.pdf. Accessed October 16, 2018.

29. Ministry of Health (MOH). Roadmap for Accelerating Reduction of Maternal and Neonatal Mortality and Morbidity in Uganda, 2007 2015. Kampala, Uganda: $\mathrm{MOH} ;$ 2007. http://library.health.go. ug/publications/service-delivery/sexual-and-reproductive-health/ roadmap-accelerating-reduction-maternal. Accessed October 16, 2018.

30. Central Statistical Office (CSO), Ministry of Health (MOH), ICF International. Zambia Demographic and Health Survey 2013-2014. Rockville, MD, USA: CSO, MOH, and ICF International; 2014. https://www.dhsprogram.com/pubs/pdf/fr304/fr304.pdf. Accessed October 16, 2018.

31. Ministry of Health (MOH), Ministry of Community Development Mother and Child Health (MCDMCH). Roadmap for Accelerating Reduction of Maternal Newborn and Child Mortality, 2013-2016. Lusaka, Zambia: MOH and MCDMCH; 2013. https://extranet.who. int/nutrition/gina/en/node/23812. Accessed October 16, 2018.

32. Serbanescu F, Clark TA, Goodwin M, et al. Saving Mothers, Giving Life Working Group. Impact of the Saving Mothers, Giving Life approach on decreasing maternal and perinatal deaths in Uganda and Zambia. Glob Health Sci Pract. 2019;7(suppl 1):S27-S47. CrossRef

33. Central Statistical Office (CSO), Ministry of Health (MOH). Zambia 2010 Census of Population and Housing Population Summary Report. Lusaka, Zambia: CSO and MOH; 2012. http://www.mcaz. gov.zm/wp-content/uploads/2014/10/2010-Census-ofPopulation-Summary-Report.pdf. Accessed November 1, 2018.

34. Central Statistical Office (CSO), Ministry of Health (MOH). Zambia 2010 Census of Population and Housing National Analytical Report. Lusaka, Zambia: CSO and MOH; 2012. https://www.zamstats. gov.zm/phocadownload/2010_Census/2010\%20Census\%20of\% 20Population\%20National\%20Analytical\%20Report.pdf. Accessed November 1, 2018. 
35. Averting Maternal Death and Disability Program. Needs Assessment of Emergency Obstetric and Newborn Care: Data Collection Modules. New York: Columbia University; 2010. https://www. k4health.org/sites/default/files/AMDD\%20NA\%20Data\% 20Collection\%20Modules.pdf. Accessed October 16, 2018.

36. Saving Mothers, Giving Life. External Evaluation of Saving Mothers, Giving Life: Final Report. New York: Columbia University; 2013. http://savingmothersgivinglife.org/docs/SMGL-Columbia-finalreport-Oct-2013.pdf. Accessed October 16, 2018.

37. American Academy of Pediatrics (AAP). Helping Babies Breathe. Itasca, IL: AAP; 2011. https://www.aap.org/en-us/advocacy-andpolicy/aap-health-initiatives/helping-babies-survive/Pages/ Helping-Babies-Breathe.aspx. Accessed October 16, 2018.

38. Lawn J, McCarthy BJ, Ross SR. The Healthy Newborn: A Reference Manual for Program Managers. Atlanta, GA: U.S. Centers for Disease Control and Prevention, 2002. Accessed October 16, 2018.

39. World Health Organization (WHO). Fact Sheet: Skilled Birth Attendants. Geneva: WHO; 2008. http://www.who.int/maternal_ child_adolescent/events/2008/mdg5/factsheet_sba.pdf. Accessed October 16, 2018.

40. Henry EG, Thea DM, Hamer DH, et al. The impact of a multi-leve maternal health programme on facility delivery and capacity for emergency obstetric care in Zambia. Glob Public Health. 2018:13 (10): 1481-1494. CrossRef. Medline

41. Kerber KJ, de Graft-Johnson JE, Bhutta ZA, Okong P, Starrs A, Lawn JE. Continuum of care for maternal, newborn, and child health: from slogan to service delivery. Lancet. 2007;370(9595):1358-1369. CrossRef. Medline

42. Lawn JE, Tinker A, Munjanja SP, Cousens S. Where is maternal and child health now? Lancet. 2006;368(9546):1474-1477. CrossRef. Medline

43. World Health Organization (WHO). Packages of Interventions for Family Planning, Safe Abortion Care, Maternal, Newborn and Child Health. Geneva: WHO; 2010. http://apps.who.int/iris/handle/ 10665/70428. Accessed October 16, 2018.

44. Bhutta ZA, Das JK, Bahl R, et al; Lancet Newborn Interventions Review Group; Lancet Every Newborn Study Group. Can available interventions end preventable deaths in mothers, newborn babies, and stillbirths, and at what cost? Lancet. 2014;384(9940):347-370. CrossRef. Medline

45. World Health Organization (WHO), Partnership for Maternal, Newborn, and Child Health. Consultation on Improving Measurement of the Quality of Maternal, Newborn and Child Care in Health Facilities. Geneva: WHO; 2014. http://apps.who.int/iris/ bitstream/handle/10665/128206/9789241507417_eng.pdf? sequence $=1$. Accessed October 16, 2018.

46. World Health Organization (WHO). Global Strategy on Human Resources for Health: Workforce 2030. Geneva: WHO; 2016. http://www.who.int/hrh/resources/pub globstrathrh-2030/en/. Accessed October 16, 2018.

47. World Health Organization (WHO), U.S. President's Emergency Plan for AIDS Relief (PEPFAR), Joint United Nations Programme on HIV/AIDS. Task Shifting: Rational Redistribution of Tasks Among Health Workforce Teams: Global Recommendations and Guidelines. Geneva: WHO; 2008. http://apps.who.int/iris/handle/10665/ 43821. Accessed October 16, 2018.

48. Basinga P, Gertler PJ, Binagwaho A, Soucat ALB, Sturdy J, Vermeersch CMJ. Effect on maternal and child health services in Rwanda of payment to primary health-care providers for performance: an impact evaluation. Lancet. 2011;377(9775):1421-1428. CrossRef. Medline

49. Ministry of Health (MOH), United Nations Population Fund. Reducing Maternal Mortality in Morocco: Sharing Experience and Sustaining Progress. Rabat, Morocco: $M O H ; 2011$. http://www. who.int/pmnch/media/membernews/2011/policy_brief_english. pdf. Accessed October 16, 2018.

50. Saving Mothers, Giving Life. External Evaluation of Saving Mothers, Giving Life: Interim Report, Phase 1. New York: Columbia University; 2013 http://www.savingmothersgivinglife.org/ docs/smgl-columbia-interim-report.pdf. Accessed October 16, 2018.

51. Davis D, O'Brien MAT, Freemantle N, Wolf FM, Mazmanian P, Taylor-Vaisey A. Impact of formal continuing medical education: do conferences, workshops, rounds, and other traditional continuing education activities change physician behavior or health care outcomes? JAMA. 1999;282(9):867-874. CrossRef. Medline

52. Mack HG, Golnik KC, Murray N, Filipe HP. Models for implementing continuing professional development programs in low-resource settings. MedEdPublish. 2017;6(1)18. CrossRef

53. Manzi A, Hirschhorn LR, Sherr K, Chirwa C, Baynes C, AwoonorWilliams JK. Mentorship and coaching to support strengthening healthcare systems: lessons learned across the five Population Health Implementation and Training partnership projects in sub-Saharan Africa. BMC Health Serv Res. 2017;17(suppl 3):831. CrossRef. Medline

54. University of California, San Francisco (UCSF) Institute for Global Health Sciences. Star Trek-inspired virtual mentor wins saving lives at birth seed grant. UCSF Institute for Global Health Sciences website. https://globalhealthsciences.ucsf.edu/news/star-trek-inspiredvirtual-mentor-wins-saving-lives-birth-seed-grant. Published August 2, 2018. Accessed October 16, 2018.

55. Aga Khan University (AKU). AKU Faculty Virtual Mentorship Programme. Aga Khan University website. https://www.aku.edu/ $\mathrm{qtl} /$ programmes/Pages/virtual-mentorship.aspx. Accessed October 16, 2018.

\section{Peer Reviewed}

Received: July 23, 2018; Accepted: September 21, 2018

Cite this article as: Morof D, Serbanescu F, Goodwin MM, Hamer DH, Asiimwe AR, Hamomba L, et al; Saving Mothers, Giving Life Working Group. Addressing the third delay in Saving Mothers, Giving Life districts in Uganda and Zambia: Ensuring adequate and appropriate facility-based maternal and perinatal health care. Glob Health Sci Pract. 2019;7(suppl 1):S85-S103. https://doi.org/10.9745/GHSP-D-18-00272

(C) Morof et al. This is an open-access article distributed under the terms of the Creative Commons Attribution 4.0 International License (CC BY 4.0), which permits unrestricted use, distribution, and reproduction in any medium, provided the original author and source are properly cited. To view a copy of the license, visit http://creativecommons.org/licenses/by/4.0/. When linking to this article, please use the following permanent link: https:// doi.org/10.9745/GHSP-D-18-00272 